\title{
Efficient Use of Bi-orthogonal Wavelet Transform for Cardiac Signals
}

\author{
Arpit Sharma \\ Student, Dept. of ECE, \\ KITE,Jaipur, India
}

\author{
Richa Sharma \\ Asst. Professor, Dept. of EE, \\ ACEIT,Jaipur, India
}

\author{
Sandeep Toshniwal \\ Reader \& Head, Dept. of ECE, \\ KITE,Jaipur, India
}

\begin{abstract}
In the detection of various cardiac abnormalities the ECG finds its importance. ECG signal de-noising process in an embedded platform is a challenge which has to deal with several issues. Noise reduction in low amplitude ECG signals by various de-noising techniques is an important task of biomedical science. ECG signals are very low frequency signals of about $0.5 \mathrm{~Hz}-100 \mathrm{~Hz}$. There are various types of artifacts which get added in ECG signals and change the original signal features, therefore there is a need of removal of these artifacts from the original ECG signal. The noises that commonly disturb the basic electrocardiogram signal are power line interference (PLI), electrode contact noise, motion artifacts, electromyography (EMG) noise and instrumentation noise. These noises can be classified according to their frequency content. In this paper, the wavelet transform based approach for removing noise is used. In this paper, the discrete wavelet transform (DWT) at level 8 was applied to noisy ECG signals and decomposition of these ECG signals was performed. After removal of noise component using thresholding technique, decomposed signal is again reconstructed using Inverse discrete wavelet transform (IDWT). Here for de-noising the ECG signal, bi-orthogonal wavelet transform is used and the most efficient idea for noise removal process is concluded with this wavelet transform. The simulation has been done in MATLAB environment with the help of SIMULINK. The experiments are carried out on MIT-BIH database. Performance analysis was performed by evaluating Mean Square Error (MSE), Signal-to-noise ratio (SNR), Peak Signal-to-noise ratio (PSNR) and visual inspection over the de-noised signal from each algorithm.
\end{abstract}

\section{Keywords}

ECG, Wavelet Transform, Discrete Wavelet Transform, MSE, PSNR.

\section{INTRODUCTION}

In biomedical signal processing, the main problem is noise which gets added in the physiological signals. In ECG various type of noise gets mixed. These are due to power line interference $(60 \mathrm{~Hz}$ or $50 \mathrm{~Hz})$, high frequency interference, external electromagnetic field, random body movements and respiration [1]. Electrocardiogram (ECG) signals are most important signals for heart condition monitoring. ECG signals gives valuable information about heart related problems in medical science by their analysis. Various types of filters have been implemented to achieve main ECG signal and to remove noisy frequency components. We can divide these filters as follows:

- Static filter or Fixed coefficients filter

- Dynamic Filter or Variable coefficients filter

It is difficult to get better result with the help of static filters in case of random noise, because we don't know the time when noise arises in signal. Due to this Adaptive filter (Dynamic filter) is most efficient for removal of noise. Fig 1 shows the standard ECG signal.

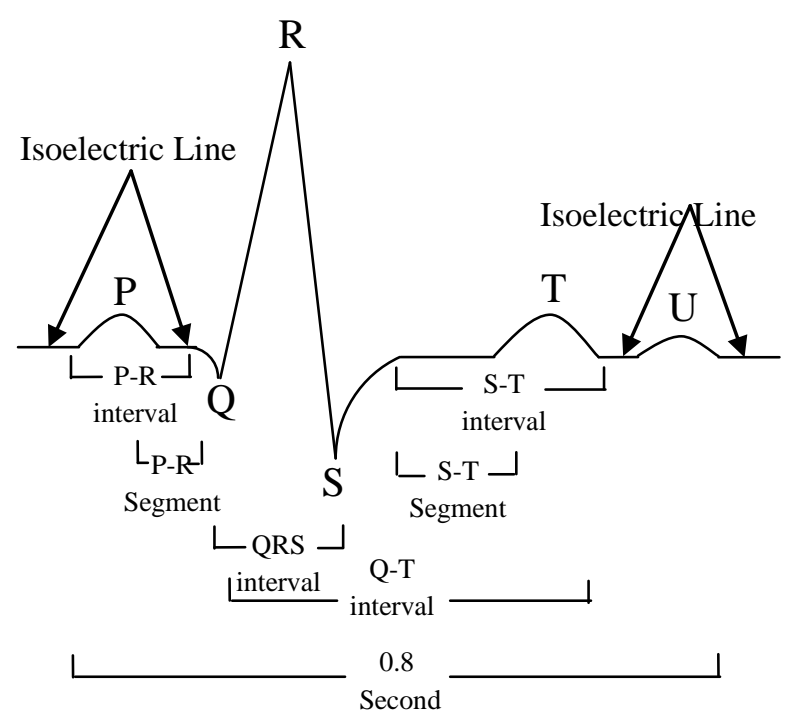

Fig 1: A standard ECG waveform

There are two sources behind interference generation as technical sources and biological sources. Technical source includes power line harmonics, high frequency noise and electromagnetic fields. While biological sources are muscular reaction $(\mathrm{EMG})$, respiratory movements, change in parameters of the direct contact between electrodes and the skin [1]. Due to this de-noising process are completely based on advanced digital signal processing in digital domain [2]. Various adaptive and non-adaptive methods are there for ECG signals de-noising or other biomedical signals (EEG, EMG) improvement [3]-[7]. For non stationary signals it is not better to use Digital static filters or Adaptive filter method because of loss of information \& low value of PSNR. The discrete wavelet transform has become a most powerful technique in biomedical signal de-noising [8]-[10].This paper utilizes the discrete wavelet transform to decompose the noisy ECG signal and then the noisy frequency components presented in the ECG were removed by thresholding process. In wavelet transform based de-noising methods threshold value is derived for removing of coefficients (details \& approximation coefficients), which are due to noise. The wavelet coefficients (details \& approximation) are derived by discrete wavelet transform of noisy ECG signal at different levels. Some wavelet coefficients have lower value. They are due to noisy components. In thresholding method we replaced them by zero (in case of hard thresholding). While higher magnitude components are due to our ECG signal which is required for signal processing. After that the de-noised signal could be reconstructed from the resulting wavelet coefficients which 
are thresholded coefficients. In recent years wavelet transform (WT) has become favorable technique in the field of signal processing. Donoho provides the important de-noising method which is called "wavelet shrinkage", there are three important steps in this method:

- Forward wavelet transform(DWT) or decomposition of signal up to Level J,

- Wavelet coefficients shrinkage at different levels by thresholding.

- Inverse wavelet transform (IDWT) or reconstruction of signal [11][12].

\section{METHODOLOGY}

Biomedical signals are low amplitude non-stationary signals. Generally biomedical signal analysis represents great resolution in time domain \& frequency domain. Wavelet analysis of signal is a windowing technique with variablesized regions for different type of signals, which is more suitable then short time Fourier transform. In Wavelet analysis for more precise low frequency information, long time intervals are used and for high frequency information, shorter regions are used. If we want processing on localized area of a larger signal (Discontinuities), wavelet has the ability to perform such task. All type of wavelet has zero average value. They can be defined for limited duration (finite duration). In Fourier analysis method we divide signal in different sine components. These components have different frequency. While in wavelet analysis, a signal is divided into shifted and scaled versions of the efficient wavelet. Wavelet transform is a Multi-Resolution analysis (MRA) technique, which is very suitable for processing of non-stationary biomedical signal [13]. In wavelet transform technique, a signal $x(t)$ which relates to the square integral subspace $L^{2}(R)$ can be defined in terms of scaling function $\left(\Phi_{j, k}(t)\right)$ and mother wavelet function $\left(\Psi_{\mathrm{j}, \mathrm{k}}(\mathrm{t})\right)$ as shown in equation (1). Here $\mathrm{j}$ represents the parameter of dilation of the visibility in frequency, while $\mathrm{k}$ represents the parameter of the position.

$x(t)=\sum_{k} a_{j o, k} \varphi_{j o, k}(t)+\sum_{j=j_{0}}^{\infty} \sum_{k} b_{j, k} \psi_{j, k}(t)$

Where coefficients a \& b according to scaling function \& mother wavelet function respectively.

\subsection{Discrete Wavelet Transform}

The discrete wavelet transform (DWT) is a type of the wavelet transform. Discrete wavelet Transform uses a discrete set of the wavelet scales. The Discrete Wavelet Transform (DWT) decomposes the biomedical signal into mutually orthogonal set of wavelets. The equation (2) and equation (3) shows scaling function $\varphi_{j, k}(n)$ and the mother wavelet function $\psi_{j, k}(n)$ respectively in discrete domain.

$$
\begin{aligned}
& \varphi_{j, k}(n)=2^{j / 2} \varphi\left(2^{j} n-k\right) \\
& \psi_{j, k}(n)=2^{j / 2} \psi\left(2^{j} n-k\right)
\end{aligned}
$$

The DWT decomposes a signal into an approximation (low frequency component) and detail (High frequency component) information. Thus the biological signal can be analyzed at different frequency components with Multi resolutions property. The DWT is achieved with the help of a pair of digital filters (HPF \& LPF). The signal is successively decomposed in DWT. Scaling function and wavelet function are corresponding with low pass and high pass filters respectively. These filters perform the decomposition of noisy
ECG signal in different frequency bands, when we apply filters recursively. Due to this decomposition process, signal is divided equally into its high frequency and low frequency components. In the DWT process, the noisy input signal $\mathrm{x}(\mathrm{t})$ is first passed through the filter bank, and the outputs of filter banks are down sampled by a factor of two for equal length. The high pass filtered signal results the detail coefficients at first level, while the low pass filtered signal results the approximation coefficients at first level. This process can continue for further level $2,3,4,5 \ldots$ Decomposition process continuous till the limit of signal length. The original signal can be reconstructed from the approximation and detail coefficients using inverse DWT.

\subsection{ECG De-noising Using DWT}

In de-noising of noisy ECG signal $\mathrm{x}(\mathrm{n})$, with this method, successively DWT \& IDWT is performed. Various types of mother wavelets are available, which have the energy spectrum concentrated around the low frequency signals. We have used Bior wavelet, which resembles the ECG wave effectively. In discrete wavelet transform approach, the low and high frequency components in noisy signal are computed by passing the signal through a filter bank (LPF \& HPF) with different cut-off frequencies. This process gives a set of approximate coefficients (cA) and detail coefficients (cD) at each level. To remove the $60 \mathrm{~Hz}$ noise and other high frequency noise, the DWT is implemented up to level 8 using bior mother wavelet function and scaling function. After that the different coefficients at level 8 are set to zero, (thresholding process) due to noisy component. Now inverse wavelet transform (IDWT) of the processed coefficients are taken to obtain the pure ECG signal. As depicted in Fig 2 the residue of the raw ECG signal and the approximate noise by components is achieved to get de-noised ECG signal.

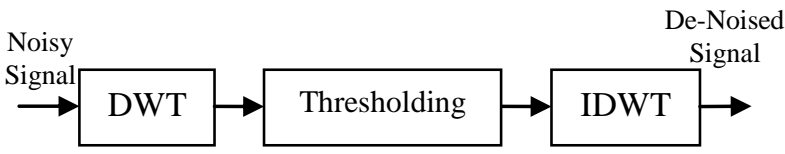

Fig 2: Wavelet Transform Based Noise Removal

\subsection{Thresholding Method}

After discrete wavelets transform process, threshold process is applied to the input signal (coefficients achieved by DWT).Global threshold value is calculated as equation (4):

$T=\sigma \sqrt{2 \log N}$

Where $\mathrm{T}$ is the threshold value, $\mathrm{N}$ is no. of samples, $\sigma$ is the standard deviation of noise for white Gaussian noise. Two thresholding methods are used namely Hard Threshold and Soft Threshold.

\subsection{Bi-orthogonal Wavelet Transform}

Bi-orthogonal wavelet shows the property of linear phase effectively. That is necessary for signal and images processing. This property is useful for decomposition \& reconstruction process in wavelet transform. In the biorthogonal wavelet transform two wavelets are used. One wavelet is for decomposition, while other for reconstruction process. Bi-orthogonal wavelet also shows the property of symmetric wavelets. We have following Bi-orthogonal wavelet filter:- bior1.1 bior1.3 bior1.5 bior2.2 bior2. 4 bior2. 6 bior 2.8 bior3. 1 bior3.3 bior3.5 bior3.7 bior3.9 bior4. 4 bior5.5 bior6.8. 


\section{RESULTS}

The ECG signals used are MIT BIH arrhythmia database ECG recording. Here base-line wander (non-stationary noise) and power line interference (stationary noise) have been considered. This MIT BIH arrhythmia database consists of two channel recording. The sampling rate for the recording is 360 samples per second. To demonstrate power line interference (PLI) cancellation all simulations are performed with MIT-BIH record number 100 . The input to the process is noisy ECG signal corresponds to the data 100 corrupted with PLI with frequency $60 \mathrm{~Hz}$. Wavelet transform was realized with support of MATLAB and Wavelet Toolbox. The ECG signals were decomposed by the DWT at level 8 . It can be observed that the activity of baseline wandering was found in the A8, since the baseline wandering is low frequency activity. In order to remove noise from the ECG signals, the synthesis process or the inverse DWT was performed. In this paper, the original signal was reconstructed with-out the A8 information. Mean square error (MSE), Peak signal to noise ratio (PSNR) and Signal to noise ratio (SNR) improvement are measured and compared. De-noising has been performed using various wavelets of Bi-orthogonal wavelet filter. In this paper Bi-orthogonal wavelet also compared with other wavelets like Daubechies, Haar, Symlet, Coif. But this paper gives conclusion that $\mathrm{Bi}$-orthogonal wavelet bior3.9 is most suitable for ECG de-noising. Here Fig 3 shows the noisy signal and the noise are removed with Bior3.9 wavelet from the original signal as depicted in Fig 4.

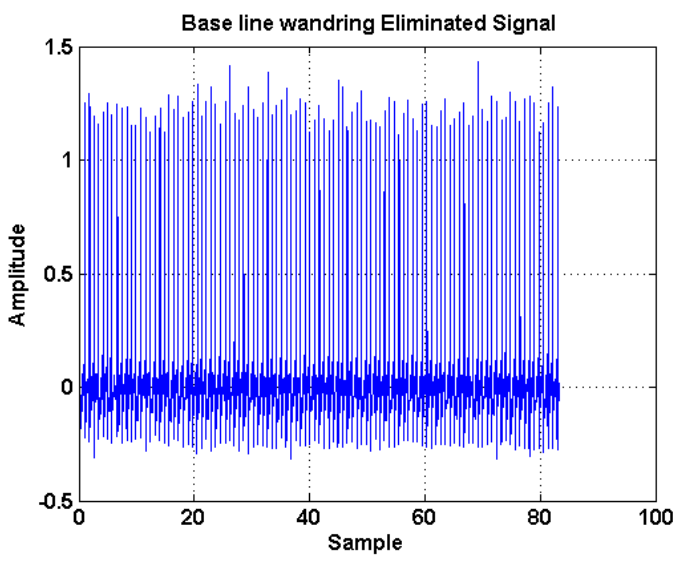

Fig 3: Noisy ECG Signal
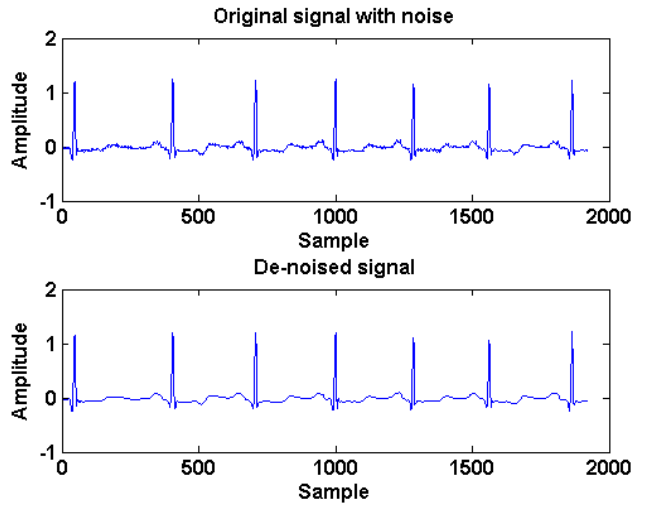

Fig 4: De-noised ECG Signal (with Bior3.9)

Fig 5 shows the four filters of Bior 3.9 by which all the filtrations are done. Fig 6 and Fig 7 shows the Frequency response of Noisy ECG signal and De-noised ECG signal respectively. Table 1 represents the calculations of signal to noise ratio with different wavelets. Fig 8 is showing wavelet and scaling function for Bior 3.9.
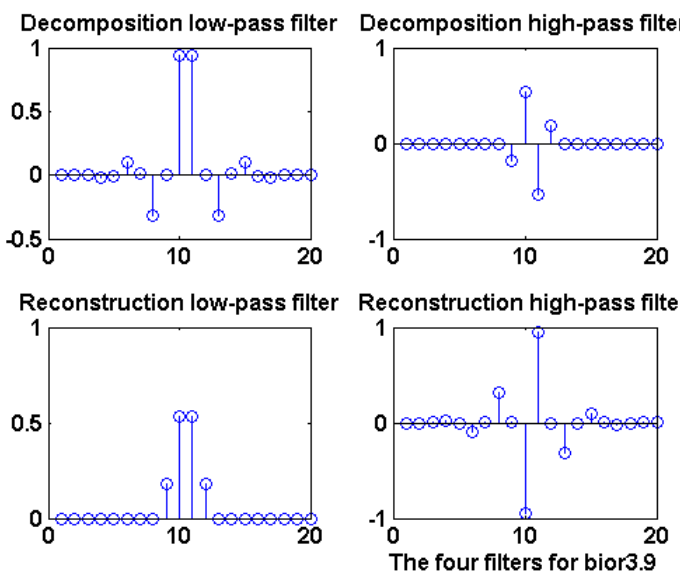

Fig 5: Bior3.9 Bi-orthogonal Four Filters

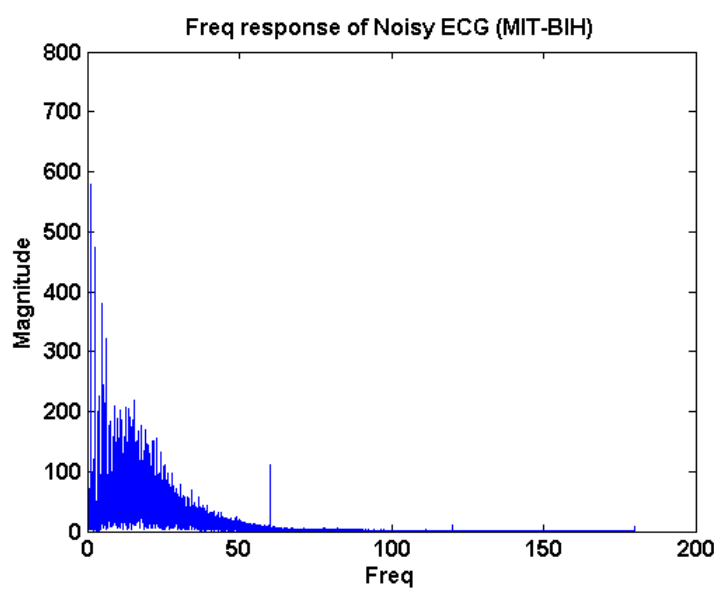

Fig 6: Freq Response of Noisy ECG Signal

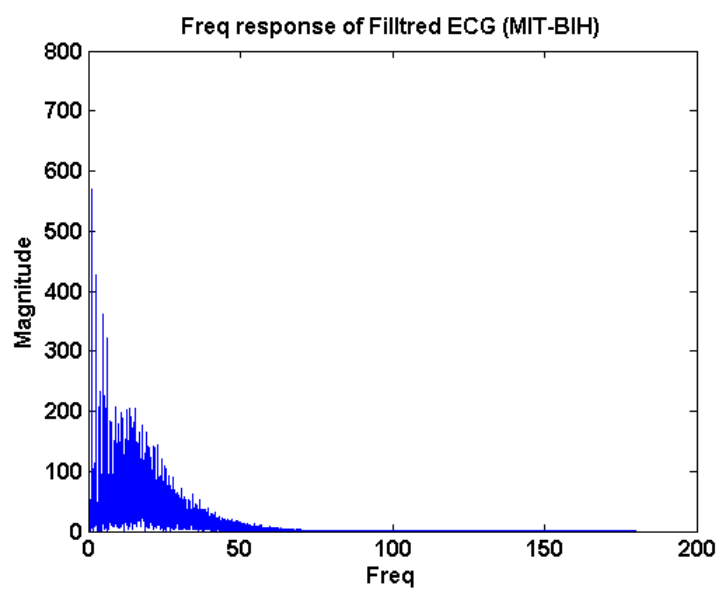

Fig 7: Freq Response of De-noised ECG (with Bior3.9) 


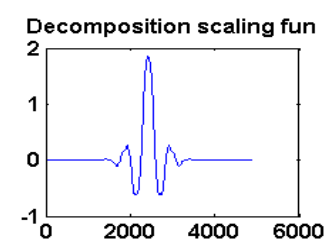

Reconstruction scaling fun
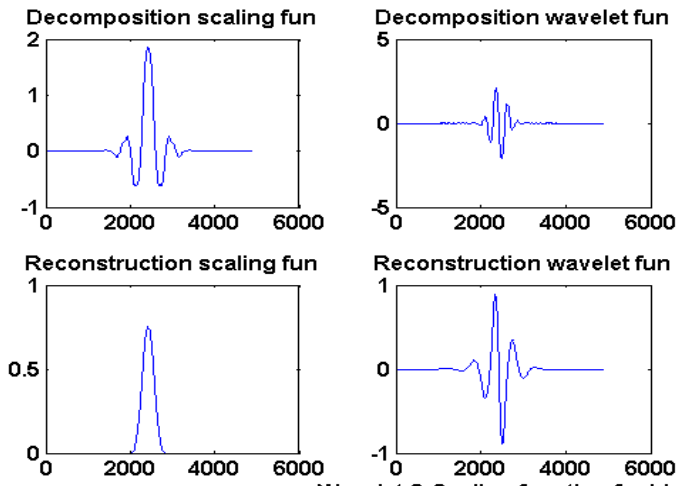

Reconstruction wavelet fun

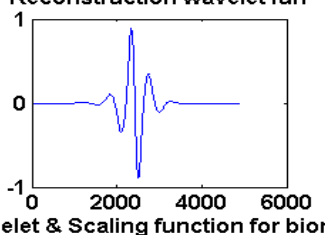

As it is seen from Fig 8, the above two figures are showing the decomposing scaling function and decomposition wavelet function, while below two are for reconstruction purpose. As it is explained earlier by using two wavelets, one for decomposition and the other for reconstruction purpose instead of the same single one, interesting properties are derived. As it is shown in Table 1 that Bior 3.9 gives the minimum Mean Square Error and the Signal to Noise ratio is maximum among all the wavelets. That's why it is more preferable.

Fig 8: Wavelet and scaling function for Bior3.9

Table 1. Performance Analysis Table for Various WAVELETS

\begin{tabular}{|c|c|c|c|}
\hline $\begin{array}{c}\text { TYPES OF } \\
\text { WAVELETS }\end{array}$ & MSE & PSNR (db) & SNR (db) \\
\hline Haar & $4.1085 \mathrm{e}-004$ & 36.9974 & 17.9362 \\
\hline Dmey & $3.9868 \mathrm{e}-004$ & 37.1279 & 18.6242 \\
\hline DB4 & $3.5226 \mathrm{e}-004$ & 37.6656 & 18.7346 \\
\hline Coif 2 & $3.4542 \mathrm{e}-004$ & 37.7507 & 18.7704 \\
\hline Sym 6 & $3.4186 \mathrm{e}-004$ & 37.7957 & 20.7645 \\
\hline Bior2.8 & $2.8113 \mathrm{e}-004$ & 38.6452 & 20.5785 \\
\hline Bior3.7 & $2.5705 \mathrm{e}-004$ & 39.0340 & 39.3732 \\
\hline Bior3.9 & $2.3774 \mathrm{e}-004$ & & \\
\hline
\end{tabular}

\section{CONCLUSION}

Various filtration techniques was applied for Noisy biomedical signals in several papers, but the wavelet Transform de-noising method is much better than other type of filtration, because spectrum of the noise interfere with spectrum of the ECG signal. By wavelet transform filtering biomedical signals are filtrated at some frequency levels, independent each other, whereas by classical filtration isn't possible to separate the low amplitude signal and noise. Therefore is using wavelet de-noising more useful then filtering. Bior (bior3.9) wavelet transform is the best method to de-noise the noisy ECG signals. As from Table 1, we found that wavelet de-noising using bior 3.9 wavelet gives lowest value of MSE and highest value of PSNR. So wavelet denoising, using bior 3.9 wavelet is most efficient method.

\section{REFERENCES}

[1] Anonymous, ANSI/AAMI EC11-1991, “American National Standard for Diagnostic Electrocardiographic Devices".

[2] Emmanual C. Ifeachor, Barrie W. Jervis: "Digital signal processing, a practical approach, second edition", Moscow: Yillyams(2004).

[3] Y.Der Lin and Y.Hen $\mathrm{Hu}$ "Power line interference detection and suppression in ECG signal processing",
IEEE Transactions and Biomedical engineering, vol.55, pp. 354- 357, January, 2008.

[4] N.V Thakor and Y.S.Zhu, "Application of adaptive filtering to ECG analysis: noise cancellation and arrhythmia detection", IEEE Transactions and Biomedical Engineering, vol.38, no.8, pp.785- 794, August,1991.

[5] J.M Leski and N.Henzel, "ECG baseline wander and power line interference reduction using nonlinear filter bank", Signal processing, vol.85, pp.781-793, 2005.

[6] Mahesh S Chavan, R A Agrawala, M.D. Uplane. "Digital Elliptic Filter Application for noise Reduction in ECG Signal" 4th Wseas International Conference on Electronics, Control \& Signal Processing Miami Florida USA 17-19 Nov. 2005 (pg 58-63).

[7] C.Mihov and I Dotsinsky, "Power line Interference elimination from ECG in case of non-multiplicity between the sampling rate and power line frequency", Biomedical Signal processing and control,vol.3,pp.334340, June ,2008.

[8] B. Natwong, P. Sooraksa, C. Pintavirooj, S. Bunluechokchai, and W. Ussawawongaraya, "Wavelet Entropy Analysis of the High Resolution ECG", ICIEA 
The 1st IEEE Conference on Industrial Electronics and Applications, May 2006.

[9] S. Gilberto, F. Thomas, R. Lutz, MR. Antoni, M. Markku, B. Klaus, B. Martin, and B. Gnter, "Multiresolution decomposition of the signal-averaged ECG using the Mallat approach for prediction of arrhythmic events after myocardial infarction", Journal of Electrocardiology, vol. 29,no. 3, pp. 223-234, 1996.

[10] M. Boutaa, F. Bereksi-Reguig, and S.M.A. Debbal, "ECG signal processing using multi-resolution analysis", Journal of Medical Engineering \&Technology, vol. 32, no. 6, pp. 466-478, November/December 2008.
[11] Donoho DL, Johnstone IM(1994). "Ideal spatial adaptation by wavelet shrinkage", Biometrika, Vol.81, No.3, pp. 425-455.

[12] Donoho DL(1995). "De-noising by soft-thresholding", IEEE Trans Inform Theory, Vol.14, No.3,pp. 612-627.

[13] Mahmoodabadi and S. Ahmadian, "ECG feature extraction based on multi-resolution wavelet transform", Proceedings of the IEEE 27th Annual Conference on Engineering in Medicine and Biology, pp. 3902-3905, Shanghai, China, January 2005. 